\title{
O.S.P
}

L'orientation scolaire et professionnelle

$36 / 1$ | 2007

Insertion, biographisation, éducation

\section{Médiations sociales et théâtre-récit}

Social mediation and Playback Theatre

Daniel Feldhendler

\section{OpenEdition}

Journals

Édition électronique

URL : https://journals.openedition.org/osp/1281

DOI : 10.4000/osp.1281

ISSN : 2104-3795

Éditeur

Institut national d'étude du travail et d'orientation professionnelle (INETOP)

Édition imprimée

Date de publication : 15 mars 2007

Pagination : 45-58

ISSN : 0249-6739

\section{Référence électronique}

Daniel Feldhendler, « Médiations sociales et théâtre-récit », L'orientation scolaire et professionnelle [En

ligne], 36/1 | 2007, mis en ligne le 05 mars 2010, consulté le 18 janvier 2022. URL : http://

journals.openedition.org/osp/1281 ; DOI : https://doi.org/10.4000/osp.1281

Ce document a été généré automatiquement le 18 janvier 2022.

(C) Tous droits réservés 


\title{
Médiations sociales et théâtre-récit
}

\author{
Social mediation and Playback Theatre
}

Daniel Feldhendler

\section{Une pratique venue d'ailleurs pour des réponses vitales}

1 Jonathan Fox, Jo Salas et leur compagnie de théatre développent une approche dynamique des récits de vie à partir de 1975, aux États-Unis, dans l'état de New York. La démarche constitue un instrument dialogique d'exploration de nos expériences vécues, de nos réminiscences individuelles et de nos histoires collectives au présent.

2 Cette méthode vise la représentation spontanée du vécu à travers un dispositif interactif original: des perceptions subjectives, des fragments de vie, des récits personnels exprimés par les spectateurs sont tour à tour représentés selon une dramaturgie particulière. Des acteurs restituent sur le champ et reflètent à travers le jeu ce qu'un membre de l'auditoire vient de communiquer. Un sentiment, une pensée, un moment de récit de vie d'une personne trouvent ainsi leur expression sur scène. Il y a en quelque sorte re-présentation condensée de la narration et re-jeu: d'où le choix de l'expression playback theatre pour qualifier la démarche. Ainsi, selon les contextes respectifs, les membres d'un groupe font mettre en scène l'expression de leurs subjectivités à travers des regards extérieurs. Dans ce modèle de dialogue transitif apparaît une dimension sociale, personnelle et singulière qui prend peu à peu forme et sens. La représentation spontanée de fragments de vie crée une dynamique où la narration singulière de récits personnels donne progressivement forme et sens à sa dimension collective.

3 Au cours d'une représentation, un dispositif interactionnel favorise le dialogue. Une personne, appelée " conducteur » (conductor), assume la fonction d'intermédiaire et de catalyseur entre spectateurs et acteurs. Le conducteur établit le cadre et crée les conditions favorables à l'échange. Après quelques mots d'introduction, il invite le public à exprimer un sentiment, une pensée correspondant à la situation et au vécu 
respectifs $\mathrm{du}$ moment. Ce petit moment exprimé par un spectateur trouve immédiatement son expression sur scène, selon une transposition dramaturgique spécifique portée par les acteurs. La forme couramment présentée au début d'une représentation est intitulée "sculpture fluide » (fluid). Des liens se créent peu à peu à travers ces échanges directs entre les participants et les courtes représentations spontanées sous forme de tableaux vivants. Ceux-ci induisent la narration de récits de vie. Dans la méthode, cette narration est appelée "l'histoire » (story). Après qu'une atmosphère d'ouverture et d'échanges a été créée, le conducteur invite une personne $\mathrm{du}$ public à venir faire le récit d'un moment vécu de son histoire. Conducteur et narrateur se trouvent assis dans un espace intermédiaire entre acteurs et spectateurs. L'interview se structure selon une forme très précise. Au fur et à mesure du déroulement de l'interview, le narrateur/conteur (story teller) est invité à choisir des acteurs pour représenter sa scène. Les acteurs respectivement choisis se mettent dans une position intermédiaire indiquant un état d'écoute accrue comme préparation à la prise de rôle. À la fin de l'interview, le conducteur résume les moments essentiels de la narration, puis il fait des propositions de forme de représentation. Les acteurs occupent alors la scène, en position de jeu, puis ils entrent en jeu et improvisent. Pendant cette phase, conducteur, narrateur et spectateurs suivent la représentation sans intervenir. La situation peut être représentée de multiples façons. Elle peut être parlée, mimée, portée par des objets, des tissus ou autres accessoires, avec ou sans accompagnement musical (dans la forme d'origine, une personne dans le rôle de musicien accompagne l'improvisation à l'aide d'instruments de musique). Il peut y avoir une seule ou plusieurs scènes. Lorsque la représentation touche à sa fin, les acteurs soulignent leur attention au narrateur en portant leurs regards vers lui. À ce moment, le conducteur demande au narrateur si la représentation a saisi l'esprit et l'essence de son expérience et de son récit. Celui-ci fait alors part de son vécu au cours de la représentation. Il peut également apporter des modifications à ce qu'il a vu et perçu dans le jeu. L'issue d'une scène peut être transformée. L'histoire prend un autre cours et le narrateur découvre comment son histoire personnelle aurait pu ou pourrait se dérouler autrement. Le narrateur-conteur devient producteur et co-auteur, metteur en scène de sa propre histoire de vie. Après ce premier récit, d'autres narrateurs-conteurs contribueront par leurs propres récits et par phénomène de résonance collective à la dynamique d'une représentation. Ainsi les récits de plusieurs conteurs, leurs histoires respectives transposées sur scène, vont révéler des thèmes collectifs spécifiques à l'auditoire présent.

4 Selon les contextes, au cours d'une représentation ou dans un atelier de formation, les membres d'un groupe partagent activement leur vécu personnel. Dans une situation d'atelier, de session de formation, les participants sont tour à tour acteurs et spectateurs en éveil, dialoguant et communiquant par l'intermédiaire de la narration de leurs histoires et de la représentation. Les uns et les autres se découvrent dans la parole et l'image, dans ce miroir et prisme intersubjectif de perceptions instantanées.

5 La méthode que nous désignons dans un contexte francophone par le terme de théâtrerécit ou théâtre en miroirs (Feldhendler, 2005) opère à partir d'un modèle de dialogue social constructif. Aujourd'hui une attitude nouvelle et un regain d'intérêt se manifestent dans de nombreux pays envers cette approche interactive de la communication. Pour expliquer ce phénomène, Fox $(1994,1999)$ et Salas (1998) se réfèrent plus particulièrement à des auteurs anglophones. Certains d'entre eux Gardner (1983), Bruner (1986), Sacks (1985) - sont bien connus dans les contextes 
francophones. Ces auteurs ont mis l'accent sur l'interaction sensorielle avec le monde environnant, la pensée et sa représentation par l'image, le besoin de narrativité et l'alliance du cognitif et de l'affectif dans l'esprit humain. Ainsi, Fox souligne que le playback theatre peut être apprécié pour la diversité de son approche des relations humaines et pour ses capacités particulières à donner réponse à nos préoccupations actuelles. Il mobilise de nombreux aspects de nos processus mentaux, constitutifs de mémoire et de conscience individuelle et collective.

\section{Le besoin de dire son histoire}

6 Salas (1998) insiste sur la nécessité de dire nos histoires. Elle souligne que dès notre naissance, notre vie est histoire et la narration de nos histoires nous accompagne tout au long de notre existence. Cette narration est constituée comme une dramaturgie. Nous savons que cette histoire prend sa source en un lieu défini et qu'elle commence à un moment précis. La narration se prolonge avec des développements, des péripéties, des rebonds et des surprises. Il y a un lieu, un moment où elle prend fin. Quand nous transformons nos expériences en histoires, nous trouvons un sens à ce que nous avons entrepris. Dire nos histoires aux autres nous aide à intégrer le sens de notre histoire singulière. L'élément intrinsèque de la forme et de la structure dans l'histoire peut transmuer le chaos et restaurer le sentiment d'appartenance à un monde qui fait sens et qui est signifiant, quelle que soit l'expérience vécue. Salas fait référence à Sacks, (un neurologue né en 1933 à Londres, puis établi à New York). Pour Sacks (1985), "si nous voulons savoir quelque chose d'un homme, nous nous demandons quelle est son histoire, son histoire réelle, la plus intime, car chacun d'entre nous est une biographie, une histoire, un récit singulier, qui s'élabore en permanence, de manière inconsciente, par, à travers et en nous - à travers nos perceptions, nos sentiments, nos pensées, nos actions ; et également par nos récits, nos discours. Biologiquement, physiologiquement, nous ne sommes pas tellement différents les uns des autres : historiquement, en tant que récit - chacun d'entre nous est unique. Pour être nous-même, nous devons avoir une biographie - la posséder, en reprendre possession s'il le faut. Nous devons nous "rassembler", rassembler notre drame intérieur, notre histoire intime. Un homme a besoin de ce récit intérieur continu pour conserver son identité, le soi qui le constitue » (p. 148).

7 Le playback theatre crée une nouvelle dimension. Notre travail, souligne Salas (1998), consiste à aller plus loin que ce que nous faisons normalement au quotidien pour révéler la forme, la figuration, la configuration dans toute expérience relatée, même si le sens reste peu clair dans la narration faite: "Nous donnons à ces histoires reconnaissance et dignité par le rituel et la perception esthétique. Nous créons des liens entre ces histoires et les narrateurs éprouvent ce qui les relient » (p. 113).

8 Salas insiste de façon catégorique sur le fait que deux éléments sont essentiels à l'identité de la méthode : une appréhension intuitive du sens donné à l'expérience du narrateur et une perception esthétique de l'histoire elle-même dans la représentation scénique. L'enjeu est fondamental dans la mesure où il repose sur l'affirmation et la reconnaissance de l'Autre quand un fragment de vie prend forme et sens de cette façon. 


\section{Créer un espace privilégié pour une dynamique de changement social}

9 L'actualité de la démarche prend toute sa dimension replacée dans le contexte sociétal actuel. Les enjeux pour répondre aux multiples défis de nos sociétés en mutation se précisent. Une idée clé fait son chemin : créer du lien social entre individu et société, en réponse aux phénomènes alarmants de délitement et de fragmentation sociale. Parallèlement, la question centrale et essentielle au devenir humain se pose : quelle éducation pour quelle humanité ? Ce questionnement s'inscrit dans une dynamique être en devenir tout en restant sujet de son histoire et créateur de sa vie, sujet agissant, non assujetti aux contingences et aux contraintes sociales et sociétales dans les processus actuels de mondialisation. Pour mettre en acte cette pensée émancipatrice et génératrice de transformations profondes, les pratiques interdisciplinaires de récits de vie s'avèrent riches en potentialités. L'approche biographique oriente le regard sur le quotidien, l'individuel et le collectif. Elle aborde nos phénomènes de société en se constituant comme moyen de représentation, de mise en forme et de structuration des trajectoires de vie (DeloryMomberger, 2000, 2001, 2003). Elle se donne pour tâches de donner du sens au vécu et de le rendre signifiant dans la recherche identitaire: « les sociétés ne s'autonomisent qu'en construisant leur histoire » (Pineau \& Le Grand, 2002, p. 119).

10 L'éclairage théorique herméneutique développé par Ricœur constitue une structure référentielle pour les praticiens francophones des récits et histoires de vie. Ricœur aborde à plusieurs reprises dans son œuvre l'importance de la fonction narrative pour l'élaboration de l'identité du sujet humain. Il constate " qu'individu et communauté se constituent dans leur identité en recevant tels récits qui deviennent pour l'un comme pour l'autre leur histoire effective » (Ricœur, 1985, p. 356). Être historien et conteur de sa propre vie introduit une dialectique particulière : le sujet apparaît comme lecteur et scripteur et il se reconnaît dans l'histoire qu'il se raconte à lui-même, sur lui-même. L'histoire d'une vie, sa figuration, ne cesserait d'être refigurée par la narration et cette activité est susceptible d'induire des changements d'ordre qualitatif. La refiguration constante de sa propre histoire dans une transformation progressive conduirait à une identité narrative. La narration active la prise de conscience de soi et de l'Autre et la connaissance de Soi-même comme un Autre (Ricœur, 1990), donc de son altérité. Celle-ci est à considérer comme facteur dynamique et structurant dans la recherche identitaire.

11 Ce concept d'identité narrative me semble être opérateur et applicable à la méthode de playback theatre dans la mesure où il désigne la configuration de l'action apportée par le récit du narrateur, comme «refondation de la valeur productrice (poḯtique) du récit pour re-présenter (mimesis) l'action en la transformant par une mise en ordre, en sens, en intrigue (muthos) » (Pineau \& Le Grand, 2002, p. 79). Par ailleurs, comme le constate Ricœur, «la notion d'identité narrative montre encore sa fécondité en ceci qu'elle s'applique aussi bien à la communauté qu'à l'individu » (Ricœur, 1985, p. 356); c'est « la sorte d'identité que le sujet humain atteint par la médiation de la fonction narrative » (Ricœur, 1988, p. 287). La médiation spéculaire, présente dans la méthode de playback theatre, ajoute à ces considérations une dialectique appréciable. Sa philosophie réside dans cet enjeu essentiel : traduire sur scène par images les propos d'un narrateur, sous une forme métonymique et métaphorique, par procédés de déplacement et de condensation, transposer et restituer l'essence et la quintessence de 
ce qui a été exprimé. En d'autres termes, les récepteurs (acteurs, musicien, et conducteur/metteur en scène) doivent saisir le sens propre et figuré d'un message, ses connotations, et lui donner une figuration signifiante aussi authentique, congruente et empathique que possible, de sorte que l'émetteur de la narration puisse recevoir, voir, entendre, découvrir et peut-être mieux comprendre sa propre histoire. Ceci constitue l'enjeu fondamental du développement de l'écoute sensible, de la réciprocité et de la réflexivité. Nous rejoignons l'herméneutique philosophique de Ricœur dont l'œuvre est guidée par une conviction centrale : le plus court chemin de soi à soi est la parole de l'autre.

12 La représentation spontanée de fragments de vie crée une dynamique qui catalyse le travail de reliance entre l'individuel et le social tout en invoquant l'intersubjectivité relationnelle. Au-delà de ce principe fondamental de réflexivité dans le travail de reliance entre l'individu et le groupe se dessine une synergie transformatrice associée à d'autres enjeux. Des thèmes sous-jacents apparaissent à travers la représentation de fragments de vie individuels. Un phénomène de socialisation par la mise en commun de ces fragments d'existentialité se met en place. Un ou des fils conducteurs se révèlent alors au fur et à mesure du développement du processus. La transposition sur scène se constitue comme moteur et elle favorise l'échange qui s'instaure dans le groupe des personnes présentes : la dimension individuelle et personnelle de la narration ouvre la voie à la résonance collective.

13 Fox (1999) insiste particulièrement sur le cadre qui favorise l'état de compréhension mutuelle approfondie et qui peut devenir vecteur de passages et de transformations. Ce théâtre de récits de vie est porté par des structures de rituel. Turner (1990), pour qui le rituel provient du théâtre, partage avec d'autres anthropologues le point de vue que « les hommes expriment dans le rituel ce qui les touche le plus» (p. 15).

Salas (1998) fait à cet égard référence à Brook (1977) qui présente la fonction ancestrale et traditionnelle du théâtre : fournir à une communauté la structure et les formes d'une réintégration temporaire, et par extension à toutes ces communautés actuelles qui vivent le quotidien dans une fragmentation. Cette fonction du théâtre ne pourrait plus s'accomplir, le processus de fragmentation sociale étant tel que nous ne partageons plus de valeurs ni de liens communs, matrices à partir desquelles le rituel instaure le lien social. Selon Brook, les auteurs et metteurs en scène, dramaturges modernes, devraient développer une nouvelle «matrice d'unité » qui est le moment, l'instant du jeu, l'ici et maintenant partagé par les acteurs et le public, le moment de re-création de liens sociaux. Le playback theatre pourrait constituer l'espace dans lequel cette matrice d'unité serait créée. Son essence repose sur le fait que tout public, aussi hétérogène qu'il soit dans ses références culturelles et sociales, partage un élément commun : le moment de la narration et la scène de la représentation provenant de la vie des individus. Nous réalisons, dit Salas, un rituel qui correspond aux besoins immédiats de l'événement et du moment : « Nous sommes orientés dans l'instant et nous participons en commun à la naissance d'une scène, la révélation de la vie, in situ, dans l'ici et maintenant » (p. 108).

Le playback theatre est donc à la fois événement artistique et événement social qui fait appel à une dynamique complexe et sensible, de communication interpersonnelle. Elle repose sur la création de cette situation qui donne sens et forme à l'expérience du narrateur à travers sa narration. La fonction spécifique des acteurs est de créer une œuvre dramatique reposant sur une compréhension profonde et accrue de la narration 
du conteur : ils transforment des moments de la vie réelle, ce "théâtre brut ", en des formes dramatiques qui vont trouver écho et résonance collective. Regarder et écouter est une expérience esthétique, une expérience d'affirmation, d'expansion et d'ouverture de soi, un moment de plaisir de mettre en partage une parole personnalisée. C'est un art qui s'engage à affirmer l'expérience de tout un chacun et à favoriser la mise en lien entre les individus de telle façon que les communautés culturelles et les groupes sociaux parcellisés puissent trouver un niveau d'échange, par la recherche d'un "plus d'humanité » dans la communication et le dialogue, essence de notre condition humaine.

L'interaction sociale portée par la représentation de récits de vie produit l'entité d'un groupe. Un tel moment privilégié peut conduire des individus à mieux entrer en relation, à s'écouter, à partager leurs vécus à travers leurs réminiscences et à exprimer pour une période déterminée un intérêt réciproque. À la base de telles relations, la notion de lien social, employée dans nos contextes francophones, pourrait s'appliquer à cette description.

17 C'est l'objet de la méthode, rappellent inlassablement leurs fondateurs, que d'être au service d'autrui. En tant qu'acte et service, elle honore la parole de chacun quelle qu'elle soit. À cette fusion peut-être unique entre forme artistique et phénomène social, s'ajoute une troisième dimension, le cadre du rituel : il a fonction catalysatrice. L'aspect cérémoniel porté par la structure et la forme de la représentation, la répartition des rôles et des fonctions avec conducteur, acteurs, conteurs/narrateurs, musicien, tout invoque présence, permanence et constance. Cette stabilité favorise la dialectique de transformation. Un tel rituel comme mise en place, en forme et en structure transmet le message symbolique que les histoires personnelles portées par ces fragments de vie ont une valeur et une qualité intrinsèques et qu'elles invitent à ce que nous $\mathrm{y}$ apportions attention et respect. Cette attitude particulière permet à l'expérience vécue de devenir narration et celle-ci trouve son prolongement dans la représentation et dans ses qualités. Les structures de rituel peuvent alors porter certaines formes de libération émotionnelle et être vecteurs de transformations personnelles et sociales.

\section{La scène, espace de médiation et de transformation}

L'espace transitionnel de la scène peut être considéré comme espace de médiation. Kaës (2002) distingue des traits constants aux figures de la médiation. La médiation comme lien transforme conjointement et corrélativement l'espace intrapsychique et l'espace intersubjectif. Toute médiation implique une représentation de l'origine, ou renvoie à une scène des origines. Elle s'inscrit dans une problématique des limites, des frontières et des démarcations, des filtres et des passages; elle est coextensive au processus de symbolisation qui suppose un écart. Dans l'ordre intersubjectif, la médiation est écart et passage de l'un à l'autre, à plus d'un autre. Dans ce passage, comme dans l'espace intrapsychique, surgit la question de l'origine du sujet et des liens qui le constituent, la représentation des limites entre leurs espaces respectifs, communs et partagés. La médiation permet au sujet d'explorer, sans s'y perdre, l'espace interne et l'espace externe, puis l'espace singulier et l'espace commun et partagé. Toute médiation suscite un cadre spatio-temporel. C'est dans cet espace-temps que s'inscrivent les enjeux des processus de transformation. 

constitue un puissant instrument de communication interactionnelle et devient potentiel de changement. Cet espace intermédiaire crée les conditions d'ouverture aux processus de transformation individuelle et sociale : la scène-miroir du playback theatre a les qualités d'une "autre scène ». La scène est définie comme locus nascendi par Moreno (1923, 1984), comme «espace esthétique » par Boal (1990), comme « espace potentiel » par Winnicott (1975) ou comme « autre scène » par Mannoni (1969). Il s'agit de la même scène, interprétée et nommée différemment selon les écoles, comme le constate Schützenberger (1981) dans sa définition du concept de locus nascendi, de la scène psychodramatique morénienne. Ce lieu de la vie est aussi espace-temps pour naître et renaître et il « représente, d'une certaine façon, l'autre scène freudienne, où se jouent les affects et les interactions que l'on saisit à l'état naissant, in status nascendi pour mieux les comprendre »(p. 206). L'espace transitionnel de la scène est lieu de compréhension qui se révèle aussi agent de transformation, comme lieu d'une réalité élargie, riche en potentialités transformatrices.

Ces considérations soulignent l'évidence et la nécessité de conditions particulières à créer pour mobiliser une synergie de spontanéité créatrice et libératrice d'énergie. Une telle mobilisation énergétique avec ses qualités cathartiques peut être moteur du processus de transformation. Pour créer une forte disponibilité, un cadre sécurisant s'impose, porté par les structures du rituel du playback theatre. Le principe essentiel de la méthode repose sur l'émergence d'une parole qui va trouver écho à travers sa restitution transposée et la mise en relation avec d'autres vécus. Fox (1999) invite à saisir l'essence de ce qui est dit et de ce qui est exprimé dans le non-verbal de la situation. Ceci requiert de créer les conditions favorables à l'ouverture et à la spontanéité des narrateurs, du conducteur et des acteurs. Des attitudes telles qu'écoute, congruence, présence, flexibilité, intuition et perception eidétique sont nécessaires. La qualité d'une écoute et d'une compréhension en profondeur vont de pair avec le degré de résonance des auditeurs, des narrateurs, du conducteur, des acteurs et musiciens. La restitution des récits se réalise à travers la représentation scénique - entre réel, imaginaire et symbolique dans cet espace particulier. La scène peut alors s'ouvrir aux récits de vie et à leur profondeur. Dans une telle situation, nous avons peu à peu accès à la qualité de ce qui fait l'essence humaine.

\section{Des pratiques pluridimensionnelles}

21 La modernité de la démarche repose sur un fait: elle se trouve à l'intersection de champs multidimensionnels qui abordent le cœur de nos pratiques et de nos relations sociales. La démarche décrite se trouve à la croisée de plusieurs champs : société, éducation, action culturelle, intervention sociale, art, thérapie. D'abord implantées dans les pays anglophones, puis germanophones, ces pratiques sont aujourd'hui présentes dans plus de cinquante pays. Une vue d'ensemble des différentes pratiques de cette approche révèle la flexibilité de son fonctionnement et la variété de ses domaines d'application, que ce soit comme représentation, intervention, atelier ou session de formation avec différents publics - des enfants aux adultes. Une école de formation a été créée en 1993 dans l'état de New York et d'autres écoles en étroite relation avec celle-ci se créent dans le monde. Il existe depuis 1990, une association internationale réunissant les praticiens en réseau que l'on peut consulter sur internet ${ }^{1}$. 
Après plus d'un quart de siècle de développement, le playback theatre s'est ouvert à des espaces, des contextes culturels et des pratiques très diversifiés. Il est présent sur tous les continents, dans des lieux institutionnalisés (écoles, universités, formation permanente, hôpitaux, centres de soins, entreprises, espaces culturels, centres sociaux, etc.). Il est de même présent dans les lieux d'enfermement (les prisons), dans la rue, dans les banlieues et dans les zones sensibles. La restitution de fragments d'expériences vives par la représentation scénique ouvre des potentialités. Briser la culture du silence dans de nombreuses régions du globe - lointaines ou proches - rapproche ces pratiques de celles du théâtre de l'opprimé(Boal, 2001, 2006) mais aussi du sociodrame morénien (Feldhendler, 1992 ; Fox, 1987; Wittinger, 2005), démarches de rechercheaction-intervention qui abordent plus particulièrement les aspects collectifs, axiologiques, sociaux et idéologiques des groupes en société. L'école de formation dans l'état de New York a commémoré en juillet 2002 sa dixième année d'existence en nous interpellant : notre époque n'a jamais été aussi favorable pour acquérir et mettre en pratique ces instruments de socialité.

Dans la mouvance novatrice des formes de représentation de situations existentielles, un nouveau projet intitulé Libra est en cours d'élaboration depuis 2001. Les objectifs formulés, révèlent les enjeux éthiques actuels : combattre le racisme, l'exclusion, la violence, l'oppression, les extrémismes et promouvoir dans son propre pays le dialogue entre individus et communautés. La génération fondatrice du playback theatre se penche aujourd'hui sur le développement de nouvelles pratiques qui favorisent la justice sociale, l'action sociale ainsi que la résolution des conflits - entreprises de restauration d'interaction sociale et d'humanité. Restituer le dialogue entre communautés meurtries, profondément blessées et traumatisées est le défi engagé. Pour Fox, l'art peut transformer et sauver des vies humaines, ainsi la mise en scène de la vie serait à considérer comme vecteur potentiel pour devenir acteur-citoyen (p. 214), auteur et protagoniste de sa vie, sujet de son histoire, sujet agissant sur le devenir humain et sur le cours de l'Histoire au présent.

Nous découvrons au quotidien cette notion fondamentale d'acteur social, concept à prendre dans tous les sens du terme, pour être auteur et devenir protagoniste de sa vie, acteur et co-créateur dans la transformation du monde en cours.

\section{Applications en éducation, dans la formation et l'insertion professionnelle}

25 La démarche de représentation de récits de vie s'avère être un instrument qualitatif dans le secteur de l'éducation et de la formation et elle gagnerait à être intégrée dans les domaines de l'insertion professionnelle. Ces pratiques peuvent être considérées comme enseignement novateur aux finalités d'éducation permanente ou comme « intervention sur l'état du monde, sur l'ordre ou le désordre des choses, sur le devenir physique ou mental des personnes, sur les grandes questions anthropologiques qui travaillent notre société » (Loriol, 2002, p. 9).

26 Par ailleurs, Meirieu (2002) souligne que le théâtre s'inscrit comme un moyen pédagogique de construction de la personne dans une collectivité, de retour sur soi et d'ouverture aux autres, de développement individuel et solidaire. Ceci peut se réaliser 
concrètement par la prise en compte de trois fondamentaux : constituer un espace symbolique, faire vivre la parole, instituer un collectif.

Constituer un espace symbolique.Par le symbolique, le théâtre fait entrer dans

l'intelligence, au sens où celle-ci consiste précisément à pouvoir tenir le réel à distance et à introduire des lignes de force, afin de se dégager de la précipitation quotidienne pour être en mesure de comprendre ce qui la structure, et de prendre de la distance pour mieux entrer dans un monde où la vie est relation entre des individus qui se reconnaissent chacun dans leur singularité et dans leurs parcours respectifs.

Faire vivre la parole. Le théâtre permet, par la prise de rôles, d'habiter une parole (celle d'un narrateur-conteur) et de la rendre vivante. Il vise à faire entendre la voix de l'individu qui l'a produite, c'est-à-dire à la restituer, incarnée et vivante.

Instituer un collectif. Il s'agit de l'institution d'un collectif dans lequel un groupe rencontre ensemble un certain nombre d'événements. Le caractère collectif, ritualisé, structuré de l'espace et du temps au théâtre constitue un antidote particulièrement puissant à la fragmentation de l'expérience vécue. La mise en partage d'expériences vécues ouvre la voie aux résonances collectives.

Dans un contexte pédagogique, la méthode de théâtre-récit constitue un entraînement actif à la réflexivité dans la communication et la démarche intègre différentes phases : écoute, compréhension, expression, action, interaction, rétroaction et mise en commun. Elle sert l'émergence d'attitudes relationnelles entre locuteurs et récepteurs. Mon expérience pratique de la méthode et son intégration dans mon enseignement à l'université ainsi que dans la formation permanente des enseignants confortent cette hypothèse. Ses formes spécifiques développent de nombreuses aptitudes: la capacité d'écoute et de compréhension, la réceptivité et l'expressivité, l'adéquation de la réponse donnée, la transposition d'un message avec d'autres supports (le corps, la voix, les sons, etc.), l'intégration de l'expression verbale et non verbale, l'expression de l'affectivité et des émotions, la perception de soi et des autres, l'ouverture à une situation nouvelle. La démarche permet d'aborder d'une façon sensible le quotidien, le vécu et les moments conflictuels par dramatisation de fragments, de récits et d'histoires de vie. En outre, cette méthode offre de précieux instruments de médiation. La démarche spécifique peut aussi être adaptée à différents éclairages thématiques sous une forme intégrée.

31 Dans le cadre de séminaires didactiques de formation de futurs enseignants qui se déroulent à l'université Goethe, la méthode est intégrée aux pratiques de biographie éducative (Dominicé, 2002) avec l'intitulé: Raconter l'école, biographie éducative et anthropologie culturelle. À travers les récits biographiques, la mémoire de l'école est très présente. Ces séminaires se proposent d'aborder cette mémoire vive de l'école comme moyen d'approche et de compréhension approfondie de situations qui ont marqué et qui marquent toute génération. Ainsi raconter l'école (voir Lani-Bayle, 2000) peut constituer une approche d'anthropoformation riche en potentialités. Le travail biographique en tant que démarche innovatrice se constitue comme instrument de connaissances pluridimensionnelles : cette approche de biographie éducative aborde de multiples aspects (relationnels, personnels, interpersonnels, interactionnels, intergénérationnels, transculturels, socio-historiques, etc.). La perspective actionnelle que nous développons à travers la représentation de récits biographiques nous permet d'élaborer des instruments didactiques dans la pratique et dans un travail de réflexion 
théorique. Raconter l'école est une invitation à intégrer la dimension du vécu et de l'expérience dans une situation d'apprentissage.

Dans cette même perspective, j'ai participé de 1998 à 2001 à un projet de coopération transnational, projet européen de formation continue ${ }^{2}$ intitulé Correspondances européennes: éducation, création et citoyenneté, il avait pour objet d'élaborer un projet pluriannuel de formation continue et de mise en œuvre d'un réseau de rechercheaction sur le thème des pratiques diversifiées de mobilité virtuelle.

Ce projet Comenius s'est étendu à cinq pays (Allemagne, Espagne, France, Italie, Pologne). Le travail de recherche-action-formation que nous y avons engagé nous a permis de mieux structurer les pratiques de formation au changement social et de mieux en cerner les enjeux, à savoir: le rôle de la création dans l'éducation, la pédagogie interculturelle et la prise en compte de l'hétérogénéité des langages, les changements du rapport au métier d'enseignant.

L'enjeu pédagogique du projet Comenius, projet transnational et transdisciplinaire, a été de recourir à une pluralité d'approches et de supports didactiques qui devaient tenir compte des contextes spécifiques des partenaires européens et des mutations socioprofessionnelles du monde enseignant. Les pratiques développées annoncent aussi d'autres enjeux fondamentaux. Former les enseignants à raconter l'école à travers leur histoire personnelle, à parler de leurs métiers et d'eux-mêmes à l'aide de méthodes actives, telle la représentation de récits de vie, c'est les engager à se mettre à l'écoute les uns des autres et à formuler leurs besoins et leurs désirs. C'est aussi entraîner les enseignants à utiliser des pratiques relationnelles dans leur quotidien professionnel. C'est, d'autre part, préparer les enseignants à transmettre les nouveaux enjeux de telles démarches à leurs élèves : apprendre l'écriture à la première personne, savoir entrer en relation et en dialogue avec l'Autre, découvrir la dynamique de la réflexivité. Les acteurs sociaux engagés dans de tels processus apprennent à devenir co-auteur et protagoniste de leur propre histoire.

Parallèlement à ces approches novatrices, j'introduis, en contexte universitaire, de nouvelles pratiques intégrant processus d'accompagnement et travail de représentation. Ces séminaires intitulés Coaching et autoréflexion: parcours étudiant et projet professionnel, proposent aux étudiants un travail de réflexion sur leurs parcours et sur leurs itinéraires respectifs en prenant appui sur des pratiques actives, à travers l'expression orale, écrite et différents procédés de communication relationnelle dynamique. Cette approche permet de mieux cerner les motivations de chacun dans un parcours de formation universitaire, dans sa propre biographie éducative, langagière et dans l'orientation professionnelle actuelle et à venir.

À cet effet, nous nous concentrons plus particulièrement sur l'échange et la mise en commun de certains aspects :

- Parcours éducatifs et motivations profondes dans le choix des études.

- Expériences vécues (séjours à l'étranger, échanges, stages pratiques, activités dans différents secteurs professionnels).

- Expériences d'apprentissage et situations professionnelles vécues.

- Orientations et perspectives personnelles.

- Réflexion sur le sens et la valeur du travail dans la société européenne actuelle.

Pour mieux être en mesure de comprendre la complexité et les enjeux de ces aspects, nous avons recours à différentes démarches: activités de communication, approche 
biographique dynamique, représentation de récits de vie, coaching pour le développement de stratégies personnelles(Angel \& Amar, 2005), pratiques de médiation et autres méthodes favorisant la prise de conscience et la réalisation de choix professionnels. Les participants de ce séminaire sont invités à rédiger, au cours $\mathrm{du}$ semestre, un journal de formation en tant que pratique autoréflexive. Ce séminaire s'inscrit dans le module de qualification "Stratégies de formation ».

Ce type de pratiques autoréflexives en milieu universitaire constitue une validation de l'expérience acquise: l'expérience individuelle partagée collectivement se fait savoir reconnue par les instances académiques (Lainé, 2005). En outre, il est à constater que les pratiques d'accompagnement comme support d'orientation et d'insertion professionnelle sont en fort développement dans l'enseignement supérieur de la République fédérale d'Allemagne.

La représentation de récits de vie s'ouvre aussi au domaine de l'insertion professionnelle. Depuis 2002, nous intervenons avec la compagnie de théâtre que nous avons créée en 1993 (Feldhendler, 2005) dans le cadre de projets issus d'initiatives municipales et d'associations proposant des programmes de formation et d'insertion aux personnes en situation de chômage. Des journées de sensibilisation sont organisées dans des espaces publics de la ville de Francfort. Sous le titre Sans travail-sens du travail - en marge de la société, une journée portes ouvertes vise à faire se rencontrer population active et chômeurs autour de thèmes très sensibles. Au cours de telles journées avec ateliers et représentations, nous prenons conscience d'une urgence: offrir aux chômeurs de courte ou longue durée un lieu pour dire leur dignité et être entendus dans un espace public. La représentation de récits de vie crée un espace potentiel pour dépasser l'isolement d'individus en autodépréciation ou en perte identitaire, et, tout en soulignant les aspects singuliers des histoires personnelles, la représentation devient médiation en établissant des parallèles entre les histoires partagées collectivement.

\section{Enjeux : vers une écologie sociale}

Le théâtre-récit ou playback theatre, instrument d'anthropologie dynamique (Schechner, 1985), présente des qualités heuristiques pour aller à la rencontre de l'Autre. Il sert l'apprentissage de la co-existence et le développement d'attitudes relationnelles fondamentales, telles qu'écoute sensible, empathie, introspection, implication, intuition, distanciation, présence et état de perception liminaire, flexibilité de rôle, disponibilité intellectuelle, affective et corporelle, spontanéité créatrice et intervention responsable. La dynamique réflexive catalyse le travail herméneutique d'émergence de sens et de reliance entre l'individuel et le social. En favorisant dialogue transitif et intersubjectivité relationnelle, le théâtre-récit devient pratique d'altérité vécue et lieu d'imaginaire social.

Ce théâtre interactif d'une étonnante vitalité se révèle très exigeant pour ceux qui le pratiquent. Ceux-ci sont appelés à s'exercer dans des pratiques formatives, à une démarche "compréhensive » et créatrice comme maïeutique et herméneutique de l'instantané.

La démarche invite à assumer son histoire et dans certains contextes à revisiter l'Histoire à travers nos histoires singulières, « expression de l'historicité, c'est-à-dire du 
travail qu'un individu effectue sur sa propre histoire pour tenter d'en maitriser le cours ", comme le pointe De Gaulejac (1999, p. 48), en référence à Sartre. Nous voici confrontés à l'un des enjeux supplémentaires de ce théâtre de récits de vie. Les histoires personnelles individuelles demandent à être reliées en une histoire collective comme communauté de mémoire.

Nous sommes appelés à un défi d'une autre envergure: sur les chemins d'un engagement inconditionnel à Soi et à l'Autre, dans cette recherche existentielle en quête de conscience commune et de reconnaissance partagée comme culture de la réciprocité.

\section{BIBLIOGRAPHIE}

Angel, P. \& Amar, P. (2005). Le Coaching. Paris : Presses universitaires de France.

Boal, A. (1990). Méthode Boal de théâtre et de thérapie. Paris : Ramsay.

Boal, A. (2001). Hamlet and the Baker's is : My life in Theatre and Politics. London : Routledge.

Boal, A. (2006). The Aesthetics of the Oppressed. London : Routledge.

Brook, P. (1977). L’Espace vide. Écrits sur le théâtre. Paris : Seuil.

Bruner, J. (1986). Actual Minds, Possible Worlds. Cambridge : Harvard University Press.

De Gaulejac, V. (1999). La Vie est-elle un roman ? Cultures en mouvement, 18, 46-48.

Delory-Momberger, C. (2000). Les Histoires de vie. De l'invention de soi au projet de formation. Paris :

Anthropos.

Delory-Momberger, C. (2001). Les Histoires de vie pour penser la mutation. Cultures en mouvement, $41,48-52$.

Delory-Momberger, C. (2003). Biographie et éducation. Paris : Anthropos.

Dominicé, P. (2002). L'Histoire de vie comme processus de formation. Paris : L'Harmattan.

Feldhendler, D. (1992). Psychodrama und Theater der Unterdrückten. Francfort : Nold.

Feldhendler, D. (2005). Théâtre en miroirs, l'histoire de vie mise en scène. Paris : Téraèdre.

Fox, J. (1987). The Essential Moreno : Writings on Psychodrama, Group Method, and Spontaneity. New York : Springer.

Fox, J. (1994). Acts of Service. Spontaneity, Commitment, Tradition, in the Non Scripted Theatre. New Paltz : Tusitala Publishing.

Fox, J. \& Dauber, H. (1999). Gathering Voices, essays on playback theatre. New Paltz : Tusitala Publishing.

Gardner, H. (1983). Frames of Mind. New York : Basic Books.

Kaës, R. (2002). Médiation, analyse transitionnelle et formations intermédiaires. In B. Chouvier et al. (éd.), Les Processus psychiques de la médiation (pp. 11-28). Paris : Dunod. 
Lainé, A. (2005). VAE, quand l'expérience se fait savoir. L'accompagnement en validation des acquis. Ramonville, France : Éres.

Lani-Bayle, M. (éd.) (2000). Raconter l'école, au cours du siècle... Paris : L'Harmattan.

Loriol, J.-P. (2002). Continuités et ruptures. In J.-C. Lallias, J. Lassalle, \& J.-P. Loriol (éd.), Le Théâtre et l'école (pp. 7-11). Cahiers n 11 . Arles : Actes Sud.

Mannoni, O. (1969). Clefs pour l'Imaginaire ou l'Autre scène. Paris : Seuil.

Meirieu, P. (2002). Le Théâtre et l'école : éléments pour une histoire, repères pour un avenir. In J.C. Lallias, J. Lassalle, \& J.-P. Loriol (éd.), Le Théâtre et l'école (pp. 30-48). Cahiers n 11 . Arles : Actes Sud.

Moreno, J.-L. (1923). Das Stegreiftheater. Potsdam : Gustav Kiepenheuer.

Moreno, J.-L. (1984). Théâtre de la spontanéité. Paris : Desclée de Brouwer.

Pineau, G. \& Le Grand, J.-L. (2002). Les Histoires de vie. Paris : Presses universitaires de France.

Ricœur, P. (1985). Temps et récit : t. 3. Paris : Seuil.

Ricœur, P. (1988). L'Identité narrative. In P. Bühler \& J. F. Habermacher (éd.), La

Narration (pp. 287-300). Genève : Labor et Fides.

Ricœur, P. (1990). Soi-même comme un Autre. Paris : Seuil.

Sacks, O. (1985). L'Homme qui prenait sa femme pour un chapeau. Paris : Seuil.

Salas, J. (1998). Improvising Real Life. Personal Story in Playback Theatre. New Paltz : Tusitala Publishing.

Schechner, R. (1985). Between Theater and Anthropology. Philadelphia : University of Pennsylvania Press.

Schützenberger, A. A. (1981). Vocabulaire de base de sciences humaines. Paris : Épi éditeurs.

Schützenberger, A. A. (2003). Le Psychodrame. Paris : Payot.

Turner, V. W. (1990). Le Phénomène rituel. Paris : Presses universitaires de France.

Winnicott, D. W. (1975). Jeu et réalité. L'espace potentiel. Paris : Gallimard.

Wittinger, T. (éd.) (2005). Handbuch Soziodrama. Die ganze Welt auf der Bühne. Wiesbaden : Verlag für Sozialwissenschaften.

\section{NOTES}

1. www.playbacknet.org

2. Programme Socrates, Comenius Action 3.1-2. 


\section{RÉSUMÉS}

Comment développer des formes de lien social qui prennent en compte les singularités et l'expression des individus en catalysant une dynamique de médiation comme reliance, mise en relation et synergie entre l'individuel et le social ? La démarche présentée dans cet article peut apporter des réponses fructueuses à ces questions: elle favorise la création d'un espace privilégié, un lieu pour dire son histoire et la relier à celle des autres. Le recours à la scène catalyse la mise en relation d'expériences singulières. Nous abordons ici un théâtre de récits de vie pour se dire et se voir, un théâtre pour devenir acteur et sujet de son histoire. La représentation de nos histoires de vie est anthropologie dynamique et herméneutique transculturelle. Elle s'ouvre à un imaginaire social riche en dynamique transformatrice.

How can one develop the kinds of social bonds which take into account the singularities and selfexpression of the individual, while at the same time acting as catalyst for a dynamic of mediation, focusing on reliance, relation building and synergy between the individual and the society? The process presented can bring fruitful answers to these questions. It allows the creation of a privileged space, a place to tell one's own story and see it in relation to those of others. To use a theatrical form catalyses the process of putting personal experience into context. Our approach here is that of a theatre where everyone can tell their personal life story and relate to that of the others; a theatre where we become both agent and subject of our story. The playing out of our life stories is both dynamic anthropology as well as cross cultural hermeneutic, and opens up a social imagination rich in transformative dynamic.

\section{INDEX}

Keywords : Dramatization, Life stories, Mediation, Memory and collective history, Social actor, Symbolic communication

Mots-clés : Acteur social, Communication symbolique, Dramatisation, Médiation, Mémoire et histoire collective, Récits de vie

\section{AUTEUR}

\section{DANIEL FELDHENDLER}

Daniel Feldhendler est enseignant-chercheur à l'université Goethe de Francfort/

Main (Grüneburgplatz 1, D-60629 Frankfurt am Main). Il participe à la formation des enseignants et intervient comme formateur dans l'éducation permanente des pays de l'Union européenne. Il est membre de l'Association internationale des histoires de vie en formation (ASIHVIF) et de l'Association internationale de théâtre de récits de vie (IPTN). Courriel : feldhendler@em.unifrankfurt.de 